\title{
Introduction: microsurgical and endovascular management of intracranial dural arteriovenous fistula
}

\author{
Gregory J. Zipfel, MD, ${ }^{1}$ David M. Hasan, MD, ${ }^{2}$ Felipe C. Albuquerque, MD, ${ }^{3}$ \\ and Adam S. Arthur, MD, MPH ${ }^{4}$
}

\begin{abstract}
${ }^{1}$ Department of Neurosurgery, Washington University School of Medicine, St. Louis, Missouri; ${ }^{2}$ Department of Neurosurgery, University of lowa, lowa City, lowa; ' ${ }^{3}$ Department of Neurosurgery, Barrow Neurological Institute, Phoenix, Arizona; and ${ }^{4}$ Department of Neurosurgery, University of Tennessee, Memphis, Tennessee

Over the past decade substantial advances in diagnostic imaging, classification, and understanding the natural history of intracranial dural arteriovenous fistula (dAVF) have been made. Paralleling these improvements in patient evaluation and risk assessment have been considerable innovations and refinements in the microsurgical and endovascular techniques by which appropriately selected patients with dAVF are treated. On the microsurgical front, minimally invasive surgical approaches with less soft tissue and bony disruption, along with enhanced tools for the intraoperative assessment of vascular anatomy and completeness of dAVF obliteration, are now commonly utilized. On the endovascular front, liquid embolic agents, balloons, and flow-directed catheters have transformed our capacity to safely and effectively treat dAVFs with a variety of anatomic configurations and locations. Innovative combinations of microsurgical and endovascular approaches are even being applied to select cases. In this issue of Neurosurgical Focus, we present a series of narrated videos that demonstrate the decision-making, vascular anatomy, and technical nuances of many of these advanced techniques, while also providing narrated videos demonstrating tried-and-true microsurgical and endovascular approaches that have proven highly effective over the years. We hope this video supplement provides a meaningful update and demonstration of modern microsurgical and endovascular approaches to patients with dAVF and aids all of us in our unending quest to provide even better care for our patients in the future. We thank the authors for their outstanding contributions.
\end{abstract}

KEYWORDS endovascular; microsurgical; technique; intracranial dural arteriovenous fistula; dAVF; video 